\title{
Reflets
}

Revue d'intervention sociale et communautaire

\section{Politiques contre le harcèlement sexuel. Comparaison et perception des agents et des plaignantes}

\section{Natalie Dupont et Christiane Bernier}

Volume 9, numéro 1, printemps 2003

Le genre en contexte : pratiques sociales et représentations

URI : https://id.erudit.org/iderudit/010862ar

DOI : https://doi.org/10.7202/010862ar

Aller au sommaire du numéro

Éditeur(s)

Reflets : Revue ontaroise d'intervention sociale et communautaire

ISSN

1203-4576 (imprimé)

1712-8498 (numérique)

Découvrir la revue

Citer cet article

Dupont, N. \& Bernier, C. (2003). Politiques contre le harcèlement sexuel.

Comparaison et perception des agents et des plaignantes. Reflets, 9(1), 53-79.

https://doi.org/10.7202/010862ar

Tous droits réservés (C) Reflets : Revue ontaroise d'intervention sociale et communautaire, 2002
Ce document est protégé par la loi sur le droit d'auteur. L'utilisation des services d'Érudit (y compris la reproduction) est assujettie à sa politique d'utilisation que vous pouvez consulter en ligne.

https://apropos.erudit.org/fr/usagers/politique-dutilisation/ 


\title{
Politiques contre le harcèlement sexuel. Comparaison et perception des agents et des plaignantes ${ }^{1}$
}

\author{
Natalie Dupont \\ Direction de la politique et de l'analyse selon les sexes \\ Développement des ressources humaines Canada \\ Christiane Bernier \\ Département de sociologie, Université Laurentienne
}

\section{Introduction}

La reconnaissance du harcèlement sexuel en milieu de travail comme problème social est assez récente. Autrefois qualifié « d'invisible " en raison des nombreuses lacunes sur la compréhension du sujet et du fait que le phénomène ne possédait pas de nom comme tel (Weeks, Boles, Garbin et Blount 1988 :431;444;449), le harcèlement sexuel est aujourd'hui devenu l'objet d'intérêt de plusieurs chercheurs et chercheures et un enjeu pour les tribunaux canadiens et américains (Lippel et Demers 1998 :9). De nombreux ouvrages se sont attardés non seulement à définir le problème, ses nombreuses composantes et ses différentes formes, mais ils ont, de plus, fait valoir le besoin de développer des stratégies pour en contrer les incidents en milieu de travail. Ainsi, depuis une quinzaine d'années, la stratégie privilégiée par les entreprises a consisté à instaurer une politique spécifique contre le harcèlement ou les manifestations de sexisme en milieu de travail et à en confier 
«Malgré la présence de politiques, le harcèlement sexuel en milieu de travail persiste.»

"L'écart important entre la prévalence $d u$ phénomène et le nombre de plaintes formelles déposées est problématique..." la gestion à des agents des plaintes. Or, malgré la présence de ces politiques, le harcèlement sexuel en milieu de travail persiste. Encore récemment, des recherches ont nettement fait voir que ce type de harcèlement existe réellement en milieu de travail, que les taux en sont relativement élevés et que ce sont les femmes qui en demeurent les principales cibles (Welsh et Nierobisz 1997; Crocker et Kalemba 1999; Dupuis, 1998; Welsh, Dawson et Griffiths 1999a). Plusieurs études révèlent aussi que les femmes tendraient particulièrement à éviter leur offenseur plutôt qu'à dénoncer le harcèlement sexuel dont elles sont victimes dans les milieux de travail dominés par les hommes (Gutek et Koss 1993; Thacker 1994; Stanton 1992; Gauvin, Marcoccio et GuéretteBreau 1999; Rowe, 1990; VanRoosmalen et McDaniel, 1998). On peut donc penser que le nombre de plaintes déposées au sein des entreprises et des organismes publics par les victimes de harcèlement sexuel ne reflète pas la prévalence du phénomène.

L'écart important entre cette prévalence supposée et les plaintes formelles déposées par les victimes est problématique et sert de point de départ à cette recherche. Selon nous, en effet, cet écart soulève deux questions importantes: (1) pourquoi les politiques instaurées dans les entreprises depuis les quinze dernières années n'arrivent-elles pas à éliminer le problème et (2) pourquoi les victimes sont-elles réticentes à témoigner lorsqu'il y a incident, qu'elles en soient victimes ou témoins, si elles sont protégées par une législation ou une politique? Des réponses partielles ont été apportées à ces questions. Ces réponses, généralement, portent sur une analyse des symboliques sexistes de notre société et sur les éléments indispensables devant assurer l'efficacité des politiques ou des programmes mis en place. Mais peu de recherches cependant ont porté sur la question de la mise en application de ces politiques et notamment sur les agents qui en sont responsables.

La présente recherche a donc examiné de plus près les éléments entourant l'efficacité des politiques en vigueur dans des milieux de travail traditionnellement masculins et dans des syndicats de travail représentant ces milieux, en utilisant une approche féministe et organisationnelle - approche qui part de l'idée selon laquelle 
le harcèlement sexuel est le produit d'une société patriarcale et qu'il est, de plus, lié à la culture organisationnelle (masculine) des entreprises.

La question générale de recherche se résume ainsi : y a-t-il congruence entre les énoncés de politiques contre le harcèlement sexuel et la perception des agents responsables de leur mise en application? Afin de répondre à cette question, il a fallu procéder à deux démarches analytiques : tout d'abord, établir une comparaison entre différentes politiques d'entreprises ou de syndicats représentant des milieux de travail non traditionnel pour les femmes; ensuite, faire une analyse de discours des perceptions d'un certain nombre d'agents des plaintes afin de cerner leur vision du phénomène du harcèlement sexuel en milieu de travail. Et pour donner un éclairage encore plus large à ces deux ensembles discursifs, quatre histoires de cas de plaignantes sont présentées; à travers leur vécu, on pourra mesurer la distance entre les volontés administratives et les réalités du terrain.

Le texte qui suit est divisé en trois parties. La première est double: elle vise d'abord à faire brièvement état du contexte historique et juridique de la problématisation du harcèlement sexuel, à faire connaitre les enquêtes effectuées dans le domaine, les informations portant sur le concept d'efficacité des politiques et les quelques informations faisant état des plaintes déposées en vertu de ces politiques; puis, à présenter l'approche qui a guidé l'analyse. La deuxième partie aborde la méthode utilisée. De type qualitatif, la recherche met l'accent sur une analyse de contenu des politiques et des discours des sujets. La dernière partie livre les résultats et permet de mettre en lumière le décalage entre ces différents discours.

\section{Contexte historique et juridique}

\section{Problématisation du phénomène}

Depuis les années 1980, une série de développements législatifs, au Canada, ont mené à la reconnaissance du harcèlement sexuel comme problème social (Smith 1995; Gauvin 1991). Certains cas 
"Les chercheurs ont identifié deux formes de harcèlement sexuel : le «climat de travail empoisonnée» et le "chantage au travail».» se sont rendus à la Cour Suprême et ont fait en sorte que le harcèlement sexuel soit reconnu comme de la discrimination fondée sur le sexe et constitue une atteinte aux droits de la personne (Gauvin 1991 : 208; Gauvin et al. 1999 : 12). Suivant ces législations, le gouvernement du Canada a obligé les entreprises canadiennes sous sa juridiction à implanter une politique en matière de harcèlement sexuel (Smith 1995 : 87).

Cependant, on a vite réalisé que les définitions développées au sein de ces premières législations s'avéraient imprécises et difficilement applicables. Des chercheurs se sont alors intéressés à l'importance du contexte entourant les incidents et ont permis d'identifier deux formes de harcèlement sexuel : le " climat de travail empoisonné " et le «chantage au travail ». Ces nouvelles définitions seront retenues par les tribunaux canadiens à la suite de l'affaire Janzen, en 1989 (Drapeau 1991 : 45-48; 59-69). On parle d'un climat de travail empoisonné lorsqu'une personne est troublée dans l'exercice de son travail par des commentaires intimidants qui ont comme effet de diminuer sa productivité. Habituellement, les commentaires portent sur la personne même ou sur le groupe sexuel dont elle est issue. Par contre, il s'agit de chantage au travail ou, de harcèlement "donnant, donnant ", lorsqu'une personne en position d'autorité altère les conditions de travail de l'employé-e en lui faisant des avances sexuelles malvenues. Ce type de harcèlement sexuel ne peut donc être le fait que d'individus qui détiennent un pouvoir hiérarchique sur l'employé-e.

De leur côté, Gauvin et al. (1999) font une distinction entre harcèlement sexuel et harcèlement sexiste, car, selon ces auteures, ces deux types de harcèlement sont spécifiques à un contexte particulier qui peut déterminer la gravité de l'incident. Selon elles, le harcèlement sexiste se définit comme étant :

"...toute conduite ou abus de pouvoir se manifestant par des actes ou des gestes à connotation sexiste répétés et non désirés. Le harcèlement sexiste se manifeste entre autres par des affirmations, des allusions, des plaisanteries ou des propositions de nature sexiste désobligeantes et persistantes qui dénigrent l'individu, 
le groupe ou l'orientation sexuelle d'une personne et qui ont pour effet d'humilier ou d'exclure une personne ou un groupe de personnes " (1999: 32).

On peut voir que cette définition portant sur le genre s'apparente au "climat de travail empoisonné » lorsque appliquée en milieu de travail.

Suite à cette théorisation du phénomène, des enquêtes nationales ont été effectuées par des organismes gouvernementaux pour évaluer la prévalence du harcèlement sexuel et le traitement des plaintes. Ce fut le cas de la Commission canadienne des droits de la personne (CCDP) en 1983, de Statistique Canada en 1994 et de Condition féminine Canada en 1999. Toutes ces enquêtes contribueront à faire reconnaitre la réalité et l'étendue du phénomène: le harcèlement sexuel persiste malgré l'existence de politiques et de législations.

\section{Développement de politiques efficaces}

Suite au constat du peu d'efficacité des politiques, on en est venu assez rapidement à s'interroger sur les éléments qui doivent composer une politique efficace. Le tableau 1 présente une synthèse de ces éléments selon les analyses de Connell 1991a; Connell 1991a et b; Gibson et Piscitelli 1995; Ganzel 1998; Matusewitch 1998 et Thacker 1994. Comme le tableau l'indique, on les a divisés selon deux catégories principales: la définition proposée et l'ensemble des procédures signalées comme nécessaires. On y remarque que dans l'ensemble, les éléments constituant une politique efficace pour les différents auteures et auteurs présentent de nombreux points communs.

Par ailleurs, en plus d'avoir mis l'accent sur l'efficacité des politiques, d'autres recherches ont porté sur différents aspects liés au dépôt des plaintes, notamment au vécu de la plaignante comme telle; ainsi on exposera: les conséquences négatives pour les plaignantes après le dépôt d'une plainte (Gutek et Koss 1993); le peu de satisfaction éprouvé par les plaignantes (Terpstra et Baker 1988); et leur type de réaction au harcèlement, surtout si le 
harceleur occupe un poste d'autorité dans l'entreprise (Crocker et Kalemba 1999).

TABLEAU 1 - Synthèse des éléments qui devraient constituer une politique efficace

\begin{tabular}{|c|c|c|c|c|c|c|}
\hline & \multicolumn{6}{|c|}{ AUTEURES ET AUTEURS } \\
\hline & Ganzel & Matusewitch & $\begin{array}{l}\text { Gibson et } \\
\text { Piscitelli }\end{array}$ & Thacker & Connel & Frierson \\
\hline Déclaration de la politique & $x$ & $x$ & $x$ & & $x$ & $x$ \\
\hline \multicolumn{7}{|l|}{ DÉFINITION DU HS } \\
\hline Déf. simple & $x$ & $x$ & $x$ & $x$ & $x$ & $x$ \\
\hline Comportements prohibés & & & $x$ & & $x$ & $x$ \\
\hline Exemples situations & $x$ & $x$ & & $x$ & & $x$ \\
\hline Protection HS extérieur & & $x$ & $x$ & & & $x$ \\
\hline \multicolumn{7}{|l|}{ DESCRIPTION PROCÉDURES } \\
\hline Enquête assurée & $x$ & & $x$ & $x$ & $x$ & $x$ \\
\hline Sanctions précises & & & & & $x$ & $x$ \\
\hline Confidentialité & & $x$ & $x$ & $x$ & $x$ & \\
\hline Mesures compensatoires & & & & & $x$ & \\
\hline Protection représailles & $x$ & $x$ & $x$ & $x$ & $x$ & \\
\hline Délai raisonnable & & $x$ & & & $x$ & $x$ \\
\hline Surv. moniteurs & & & & & $x$ & \\
\hline Autres langues & & $x$ & & & & \\
\hline Resp. administratifs & & & $x$ & $x$ & $x$ & $x$ \\
\hline Choix _/_ & & $x$ & & & & \\
\hline $\begin{array}{l}\text { Coord. agents/ } \\
\text { agences fédérales }\end{array}$ & $x$ & $x$ & & $x$ & & \\
\hline
\end{tabular}




\section{Cadre d'analyse}

"L'approche féministe organisationnelle s'inscrit dans le champ féministe, mais permet de préciser les enjeux en intégrant dans l'analyse à la fois les rapports de sexe et de pouvoir et le contexte organisationnel de l'entreprise.»
Deux approches théoriques sont généralement retenues pour comprendre et expliquer le harcèlement sexuel :l'approche féministe comme telle et l'approche féministe organisationnelle. L'approche féministe apparaît le plus souvent dans les ouvrages qui associent le harcèlement sexuel à l'idéologie patriarcale, à une construction sociale du pouvoir, c'est-à-dire à une forme de violence continue exercée contre les femmes dans leur vie professionnelle et personnelle (Stanton 1991; Sev'er 1999; Savoie et Larouche 1988). Bien que nécessaire, cette approche reste néanmoins trop vague pour être appliquée directement au phénomène du harcèlement en milieu de travail typiquement masculin.

L'approche féministe organisationnelle s'inscrit dans le champ féministe, mais permet de préciser les enjeux en intégrant dans l'analyse à la fois les rapports de sexe et de pouvoir et le contexte organisationnel de l'entreprise. Elle est plus spécifique à l'étude des milieux de travail. Signalons, par exemple, le modèle développé par Sev'er (1999) dans lequel le harcèlement sexuel est perçu comme une responsabilité sociale qui renvoie à la structure sexuée de la gestion organisationnelle. Par exemple, en ce qui concerne la distribution sexuée du pouvoir hiérarchique, la composition de la main-d'oeuvre et sa répartition hommes-femmes en milieu de travail: selon Sev'er, ce sont les structures verticales de pourvoir qui contribuent aux incidents de harcèlement sexuel. Et puisque les femmes sont toujours au bas de l'échelle, elles sont plus susceptibles d'en être victimes.

\section{Méthodologie de la recherche}

L'enquête a été organisée autour de trois échantillons: les politiques d'entreprise, les agents des plaintes et quelques plaignantes.

\section{Échantillonnage et collecte des données}

L'échantillonnage des politiques s'est fait sur la base des entreprises privées qui possèdent une telle politique. Elles devaient chacune 
répondre à quatre caractéristiques: (1) être traditionnellement masculine, (2) posséder une politique en matière de harcèlement à l'intention des victimes, (3) être située dans le Nord-Est de l'Ontario et (4) comporter des employées et employés qui soient syndiqués. La région nord-est ontarienne fut sélectionnée parce qu' on y trouve un certain nombre d'entreprises traditionnellement masculines. De plus, la rareté d'études de ce genre, étant donné l'implantation relativement récente des politiques dans la plupart de ces entreprises, justifiait aussi le choix de cette région. Huit politiques ont été retenues pour comparaison (dont deux conventions collectives, deux politiques syndicales et quatre politiques issues du département des ressources humaines et l'administration de l'entreprise).

L'échantillonnage des agents des plaintes s'est effectué par téléphone. Onze représentants syndicaux et un représentant des ressources humaines - onze hommes et une femme ${ }^{2}$ - ont accepté de participer à une entrevue. Ensemble, les agents représentent six entreprises ou sections locales situées à SaultSte-Marie, Kapuskasing, Sudbury, North Bay et Timmins, et deux syndicats au niveau national et international. Malgré la diversité des tâches attribuées aux postes qu'ils occupent, les agents ont aussi la responsabilité de traiter et d'enquêter sur les plaintes qui proviennent de leur milieu de travail. La moyenne d'âge des agents est de 45,3 ans. La moitié d'entre eux possèdent un diplôme postsecondaire et un seul possède un diplôme universitaire. Le nombre d'années à leur poste est, pour la plupart, de 0 à 10 ans alors qu'un seul agent a plus de 16 ans de service. En raison des restrictions liées à la confidentialité, aucun agent n'a pu nous aider à recruter des plaignantes ce qui a eu pour effet qu' aucune n'a pu l'être pendant l'organisation de l'enquête. Mais d'autres démarches, effectuées par des comités de femmes ou des membres du réseau de la recherche, ont permis d'identifier quatre femmes qui ont déposé une plainte dans une entreprise traditionnellement masculine dans le Nord-est ontarien. 


\section{Méthode d'analyse}

Les données qui proviennent des trois échantillons ont été soumises à une analyse discursive à partir de thématiques. Les principaux thèmes dégagés suite à la catégorisation des éléments des politiques sont les suivants : (1) la mission des entreprises ou des syndicats en ce qui concerne la mise en œuvre de la politique, (2) la définition du harcèlement sexuel et (3) les procédures de plainte. Une fois l'organisation des informations complétée, l'analyse des politiques a été confrontée aux éléments théoriques qui figurent dans le tableau 1. Il a donc été possible de voir dans quelle mesure les politiques analysées comprennent les éléments identifiés par les auteures et auteurs comme nécessaires à la constitution d'une politique efficace.

Les principaux thèmes provenant des entrevues auprès des agents sont: (1) leur connaissance de la politique en matière de harcèlement sexuel tant au plan de la définition du harcèlement sexuel qu'à celui des procédures de plaintes, (2) leur perception en ce qui a trait aux effets de la politique et aux attitudes des collègues (masculins) envers les plaignantes et (3) leur évaluation des changements qui devraient être apportés à la politique et les raisons qui expliquent le peu de plaintes déposées. Et pour les plaignantes : (1) la perception des plaignantes du harcèlement sexiste, (2) les éléments dans leur discours qui s'insèrent à l'intérieur du continuum du harcèlement sexuel au harcèlement sexiste, (3) l'attitude de leurs collègues de travail pendant et après leur plainte, (4) leur degré de satisfaction par rapport à la résolution de la plainte, (5) leur évaluation de la politique contre le harcèlement sexuel et (6) leur sentiment, aujourd'hui, à l'égard du processus de plainte.

\section{Contenu des politiques et discours des agents}

\section{Comparaison des politiques}

On pourrait supposer que certains éléments constitutifs de base sont nécessaires pour créer une politique en matière de harcèlement sexuel. Une première lecture des politiques a permis 
d'identifier des catégories à partir desquelles la comparaison a été rendue possible: (1) une déclaration d'intention ferme ou un énoncé d'intolérance à l'endroit du harcèlement de la part de l'entreprise (ou du syndicat), (2) une définition exhaustive du harcèlement sexuel accompagnée d'exemples de situations et de matériel et de gestes prohibés et (3) une description élaborée des procédures de plainte.

On constate aisément que, dans l'ensemble, les politiques retenues se rapprochent du modèle (Tableau 1) en ce qui a trait à ces trois éléments de base. Premièrement, comme on pouvait s'y attendre, elles possèdent toutes un énoncé qui stipule une prise de position de l'entreprise ou du syndicat contre le harcèlement sexuel ou, du moins, un énoncé qui a trait à la défense des droits fondamentaux des travailleuses et travailleurs et à la non tolérance du harcèlement sexuel en milieu de travail. Deuxièmement, les politiques possèdent toutes une définition qui comprend une description des conduites prohibées ou des exemples de situations qui constituent du harcèlement. La moitié des politiques font aussi allusion au climat empoisonné en prohibant l'affichage du matériel pornographique en milieu de travail et en présentant des exemples de comportements et de situations qui, indirectement, y font référence. Cependant, il faut noter que les définitions des deux formes de harcèlement sexuel retenues par la jurisprudence canadienne - "climat de travail empoisonné " et harcèlement "donnant, donnant" — ne sont pas mentionnées comme telles dans ces politiques d'entreprises, pas plus, d'ailleurs qu'elles n'étaient spécifiées par les auteures et auteurs, dans le modèle de politique efficace comme on l'a vu au Tableau 1. Toutefois, les politiques y font allusion à peu près partout en interdisant les demandes d'échanges de nature sexuelle ou l'affichage de matériel pornographique. Troisièmement, en ce qui concerne les procédures de plainte, on peut dire que les politiques correspondent au modèle d'interprétation sur les points suivants : l'ouverture d'une enquête suite au dépôt d'une plainte, la description des sanctions et, bien sûr, elles s'entendent sur le respect de l'anonymat des personnes et la confidentialité des dossiers de plainte. Quoique la description des démarches à entreprendre differe d'une politique à l'autre, l'absence de procédures formelles ne signifie pas qu'une victime ne pourrait pas faire appel à une telle procédure. 
Mais au-delà de ces similitudes générales entre les politiques, moins de la moitié d'entre elles ont dépassé ce niveau et ont présenté des éléments précis et plus contraignants tels que:

(1) la spécificité des sanctions selon la gravité et la récurrence du délit - les politiques ont énoncé, sans plus, que des mesures disciplinaires appropriées seront prises si l'accusé est trouvé coupable (ce qui laisse place à beaucoup de laxisme);

(2) la gestion de la protection des plaignantes pendant l'enquête et lors du retour dans le milieu de travail. En fait, aucune politique ne mentionne l'obligation d'assurer un suivi. Cette absence nous paraît particulièrement étonnante, surtout dans le cas des politiques syndicales. Voilà sans doute l'un des éléments essentiels mis en lumière par l'analyse;

(3) la reconnaissance des effets psychologiques du harcèlement sexuel et sexiste sur la victime;

(4) la reconnaissance des effets sociaux que le harcèlement peut entraîner sur les relations de travail - diminution de la performance au travail et de la force syndicale - et sur la société dans son ensemble - marginalisation des femmes qui travaillent dans ces milieux et obstacle au développement de leur plein potentiel.

"Ces différences, relevées par l'analyse, permettent de voir que les politiques issues d'entreprises privées sont souvent mieux élaborées que celles provenant des syndicats.»
Ces différences, relevées par l'analyse, permettent de voir que les politiques issues d'entreprises privées sont souvent mieux élaborées que celles provenant des syndicats. Les deux conventions collectives, en effet, peuvent être qualifiées de «floues » à ce sujet: plusieurs des éléments qui renvoient à la définition du harcèlement sexuel, par exemple, n'ont pas été inclus; de même, les textes sont très succincts, voire non existants, en ce qui concerne les procédures de plainte. Puisqu'il s'agit de conventions collectives, on pourrait probablement expliquer cette brièveté par le fait qu'on estime que les plaintes seront traitées ailleurs, en vertu d'une autre politique, soit celle générée par le département de gestion du personnel, dans l'entreprise, ou soit encore parce que, au niveau national, le syndicat de référence a déjà une politique élaborée.

Mais en dépit des variations entre les politiques et des changements qui pourraient leur être apportés, on peut voir qu'elles concordent généralement avec le modèle construit et qu'en fait, sauf pour 
les points soulevés, elles sont relativement complètes. Le tableau 2 présente la synthèse des éléments mentionnés dans les politiques d'entreprise analysées, en fonction des trois catégories mentionnées cidessus. On peut facilement y voir les recoupements avec le Tableau 1.

TABLEAU 2 - Synthèse des éléments mentionnés dans les politiques en matière de HS

\begin{tabular}{|c|c|c|c|}
\hline CATÉGORIE & THÈME & ÉLÉMENTS MENTIONNÉS & $N=8$ \\
\hline Énoncé de mission & $\begin{array}{l}\text { Défense des droits } \\
\text { fondamentaux } \\
\text { Tolérance zéro }\end{array}$ & $\begin{array}{l}\text { - HS = atteinte aux droits de la personne } \\
\text { - simple affirmation }\end{array}$ & $\begin{array}{l}8 \\
8\end{array}$ \\
\hline $\begin{array}{l}\text { Définition du } \\
\text { harcèlement sexuel }\end{array}$ & Définition du harceleur & $\begin{array}{l}\text { - gestes prohibés (ex: regards, contacts physiques, } \\
\text { avance sexuelles) } \\
\text { - } \text { abus verbal/paroles } \\
\text { - } \text { menaces générales } \\
\text { - } \text { demandes sexuelles/sollicitation } \\
\text { - interdiction de l'affichage de matériel dégradant } \\
\text { - } \text { mauvaise performance au travail } \\
\text { - caractéristiques qualifiant le mauvais climat de travail } \\
\text { - commentaires non dirigés à une personne spécifique } \\
\text { mais à un groupe (ex: femmes) } \\
\text { - diminue respect/dignité de la personne } \\
\text { - sentiments d'humiliation } \\
\text { - diminue la bonne performance au travail } \\
\text { - relations de travail en danger } \\
\text { - sécurité de la victime affectée } \\
\text { - force syndicale affectée } \\
\text { - peut être en position d'autorité } \\
\text { - peut être un collègue de travail } \\
\text { - peut provenir de l'extérieur } \\
\text { - femmes comme cibles } \\
\text { - milieu de travail — tous les départements } \\
\text { - endroit lié au milieu de travail (ex: conférences, } \\
\text { cafétéria, activités syndicales) }\end{array}$ & $\begin{array}{l}7 \\
7 \\
6 \\
8 \\
6 \\
1 \\
1 \\
1 \\
2 \\
4 \\
3 \\
2 \\
1 \\
1 \\
7 \\
7 \\
4 \\
1 \\
7 \\
3\end{array}$ \\
\hline
\end{tabular}




\begin{tabular}{|c|c|c|c|}
\hline CATÉGORIE & THÈME & ÉLÉMENTS MENTIONNÉS & $N=8$ \\
\hline $\begin{array}{l}\text { Procédures de } \\
\text { plainte }\end{array}$ & $\begin{array}{l}\begin{array}{l}\text { Démarches par type } \\
\text { de plainte } \\
\text { informelle } \\
\text { formelle }\end{array} \\
\text { Enquête suite à la plainte } \\
\text { Sanctions } \\
\text { Retour en milieu de travail }\end{array}$ & $\begin{array}{l}\text { - description des procédures informelles } \\
\text { - description des procédure formelles } \\
\text { - énoncé général qu'une enquête sera effectuée } \\
\text { - } \text { spécificités de l'enquête (ex: préparation d'un } \\
\text { rapport, résolution } 10 \text { jours après la plainte) } \\
\text { - énoncé général sur les mesures disciplinaires } \\
\text { appropriées } \\
\text { - sanctions spécifiques allant jusqu'au renvoi } \\
\text { - spécificités (ex: référence au programme d'assistance } \\
\text { aux employés, envoie d'une lettre à la section locale) } \\
\text { - protection des plaignantes contre les représailles } \\
\text { attribuables au dépôt de plainte }\end{array}$ & $\begin{array}{l}8 \\
5 \\
8 \\
5 \\
5 \\
4 \\
4\end{array}$ \\
\hline
\end{tabular}

\section{Discours des agents des plaintes}

Les données obtenues lors des entrevues auprès des agents des plaintes ${ }^{3}$ ont été organisées en fonction des catégories suivantes : (1) les informations générales sur la politique et son implantation dans leur entreprise, (2) leur perception de l'impact de la mise en application de cette politique, de l'attitude des collègues de la victime suite au dépôt d'une plainte et de la satisfaction des plaignantes et (3) l'évaluation qu'ils font de son efficacité et des changements à y apporter et des raisons qui expliquent le peu de plaintes.

En ce qui concerne la première thématique, on a pu constater que le contexte qui entoure la genèse de l'instauration de la politique, de même que la façon de décrire les procédures utilisées pour le traitement d'une plainte sont assez semblables d'un agent à l'autre. Plus souvent qu'autrement, une politique est mise en vigueur à la demande du syndicat; selon certains, il s'agit d'une décision conjointe entre l'entreprise et le syndicat alors que les autres ont signalé qu'il s'agit d'une initiative de l'entreprise seule. La définition du harcèlement sexuel, telle que formulée par les 
"...la moitié des agents parleront de changements positifs dans leur milieu de travail...» "...mais ils font tous état de réactions manifestes de la part des employés masculins...» agents, suit d'assez près celle présentée dans leur politique. Ce qui semble le plus remarquable, cependant, dans leurs énoncés, c'est l'importance qu'ils accordent au côté interprétatif du comportement abusif du point de vue de la victime: un geste, une parole, une proposition ne signifient pas la même chose pour chacune et il faut partir de là:

I guess what actually constitutes [sexual harassment] from a legal perspective, sounds like to me it's pretty wide open in that it's really a question of how the individual on the receiving end perceives it and sees it (Ag 05).

En ce qui a trait à la deuxième thématique, la moitié des agents parleront de changements positifs ayant eu lieu dans leur milieu de travail depuis l'implantation de la politique. Ces changements ont surtout trait aux attitudes sexistes traditionnelles, c'est-à-dire aux changements de comportement des hommes en matière de rejet et de mépris des femmes:

I guess the male attitude has changed somewhat, I think part of the reason for that is policies like our harassment policy. Men are more willing to accept that women can work in the mining industry. And they can do a pretty good job of it too ( $\mathrm{AgO})$.

Trois responsables des plaintes affirmeront cependant n'avoir pas vu de changements réels.

Par contre, en ce qui a trait à l'attitude des employés de sexe masculin lors de l'implantation de la politique comme telle, les agents font tous état de réactions manifestes de la part des employés pouvant s'exprimer de diverses façons: soit que les hommes n'ont pas pris la politique au sérieux, soit, qu'au contraire, ceux-ci se sont sentis menacés par elle. Un agent a même rapporté l'exaspération des employés de son entreprise à l'égard des contraintes amenées par la politique. Les choses, selon lui, ont empiré parce que les employés sentaient qu'ils devaient réprimer leurs comportements: 
Oh! This Human Rights policy, you can't do anything anymore, you can't do this, you can't do that! (Ag01).

Un seul agent a souligné que l'effet ne fut pas si négatif si ce n'est que les hommes étaient déçus de faire disparaitre le matériel pornographique qui était affiché sur les murs :

I don't remember any back-lash to it other than the, I guess, the general disappointment that they had to take the girly nudies, the nude pictures down. I don't remember anything really, you know, anything all that negative (AgO4).

La majorité d'entre eux affirment aussi que l'ensemble des employées et employés des compagnies connait l'existence de la politique à l'intention des victimes qui désirent déposer une plainte. Ils mentionnent d'ailleurs les nombreux efforts déployés pour rendre la politique visible. Cependant, lorsque l'on soulève la question de la différence entre les hommes et les femmes au sujet de la connaissance de cette politique, la moitié des agents ont indiqué que les femmes sont plus conscientes de son existence que les hommes parce qu'elle est implantée à leur intention.Ainsi, elles seraient plus au fait des droits et des mécanismes pour les protéger lors d'incidents.

Enfin, l'attitude des collègues de travail des plaignantes et la satisfaction de ces dernières au sujet de la résolution de la plainte sont perçues comme étant positives par les agents (ce qui a été contredit dans le témoignage des quatre victimes). Mais une des personnes a ajouté qu'en dépit de la satisfaction des plaignantes, elles peuvent prendre beaucoup de temps à oublier l'incident.

On a demandé aux agents si la politique de leur entreprise leur semblait efficace et si des changements leur apparaissaient nécessaires. Bien que certains d'entre eux croient effectivement que des changements sont nécessaires, - ceux-ci parlent de transformations au niveau des procédures d'enquête ou au niveau de la définition des sanctions - on perçoit une certaine ambivalence dans leur discours: ils ne veulent surtout pas donner 
l'impression que la politique de leur entreprise n'est pas pertinente ou efficace. Par exemple:

If there was an incident where somebody was sexually harassed I don't think anyone would feel nervous about or reluctant to put in a complaint, I'm sure they would. We always, you know, as a union, we're always there to help them address their problems and keep it as confidential as possible. So I hope that if there was somebody that had a complaint that they would feel free to come forward and I think they would (Ag 08).

On se conforte en parlant du système en place, des procédures qui sont bonnes et respectées.

Le même type d'ambiguité se fait jour lorsque, après avoir reconnu que certaines transformations étaient nécessaires, quatre des agents expliquent le peu de plaintes déposées dans leur entreprise ou au syndicat par des raisons directement liées à la mise en application des politiques. Cependant, de telles raisons ne semblent pas remettre en cause la politique comme telle ou sa gestion. Au contraire, le peu de plaintes renvoie principalement, à leur avis, à des raisons relevant des victimes elles-mêmes. Le propos s'attarde soit: à la peur qu'ont les victimes de perdre leur emploi suite au dépôt d'une plainte, particulièrement pour celles qui ont un statut précaire, soit au fait qu'elles craignent pour leur sécurité ou encore pour la suite de leurs relations de travail :

It's really, it's a fairly rare occurrence. Because, you know, either the women are afraid to complain, because they'd be afraid that they won't get a summer job next year.

«... certains des agents ne croient pas que les victimes ont peur de se plaindre ou ont raison d'avoir peur...
[...] You know, so they're probably a lot more quiet about it (AgO4).

Finalement, signalons que trois des agents ne croient pas que les victimes ont peur de se plaindre ou du moins disent-ils, si c'est le cas, elles n'ont pas raison d'avoir peur, puisque, selon eux, elles sont censées connaitre leurs droits et savoir que le mécanisme est institué à leur intention. Ici, on voit très bien la différence 
entre les points de vue : certains agents tiennent compte du harcèlement de type "climat de travail empoisonné " et comprennent pourquoi les femmes ne veulent pas se plaindre, alors que d'autres ne conçoivent le harcèlement que comme un abus physique, un geste ou une parole dirigée vers une personne en particulier.

\section{Discours des plaignantes}

«...dans un esprit de vengeance, ces collègues l'ont accusée, à son tour, de harcèlement sexuel...»
Chaque histoire de cas a ses particularités et s'étend sur une période de vingt ans : deux plaintes ont été déposées à un organisme public en vertu de discrimination et non de harcèlement sexuel, alors que les autres ont été déposées (1) auprès d'un superviseur à une époque où la politique n'était pas en vigueur et (2) au ministère du Travail en vertu de la Loi sur la sécurité au travail. Les plaignantes ayant fait appel à un organisme public ont obtenu gain de cause mais elles ont dû, toutefois, défendre leur litige pendant plusieurs années. L'une d'entre elles a dû impliquer les médias afin de se faire entendre, finalement, et, l'autre, quoique l'entreprise ait été reconnue coupable de discrimination sexuelle, a été exclue de la compagnie, au bout du compte, avec une pension pour personne handicapée; en effet, entre temps, dans un esprit de vengeance, ses collègues l'ont accusée à son tour de harcèlement sexuel, et ce, en vertu d'une politique qu'elle avait elle-même aidé à promulguer. Le résultat pour les deux autres plaignantes est plus ou moins satisfaisant: une plaignante a reçu une lettre d'excuses de la part de l'entreprise qui, en conséquence, a mis en vigueur une politique en matière de harcèlement, alors que l'autre a décidé de ne pas poursuivre l'entreprise puisqu'elle n'était qu'employée temporaire.

Bien que les circonstances autour de chaque dépôt de plainte varient, la nature du traitement sexiste dont ces femmes ont été victimes ne fait pas de doute. Pourtant, elles ont attendu longtemps avant de porter plainte. Et bien qu'il s'agisse de harcèlement sur la base du sexe dans tous les cas, les femmes n'ont pas nécessairement voulu utiliser ce motif comme dépôt de plainte, afin, justement, d'en éviter les difficultés et les retombées, disent-elles: pourtant, 
elles continuent d'être victimes de harcèlement après la plainte, elles subissent une attitude négative de la part de leurs collègues masculins :

Some people were very very upset because I had registered a complaint. One foreman came to us and told us that we should be at home with our children and that's where we belonged. Some of the foremen wouldn't talk to us ever again (Nicole),

et pour une plaignante, Lynn, il s'agit d'accusations et d'une mise à l'écart comme handicapée. Elle affirme d'ailleurs amèrement : "That's my disability, my gender ».

Enfin, elles se sont toutes dites conscientes de l'existence de la politique qui prévalait dans l'entreprise mais elles affirment qu'elle n'est pas du tout efficace et la perçoivent comme un document qui, malgré la non tolérance pour le harcèlement en milieu de travail qui y est exprimée, n'est pas mis en vigueur. Une plaignante affirme que les procédures dont dispose la politique ne sont pas respectées et que les personnes responsables du traitement des plaintes ne sont pas disponibles :

...the company's policy is in the garbage, how's that? Because there's nobody that you can go to, there's no committee anymore. It's all written up, the policy is there, but who do you go to? There's no committee, there's nobody to talk to, there's nobody there that looks into it. There's no investigations. There's nothing, there's nothing...

Les plaignantes ayant eu recours à un organisme public pour se plaindre n'ont pas peur d'exprimer leur vision par rapport au processus utilisé. Par exemple :

They never talked to the union, they never went after the union, all the things that they said they were going to do, or could do, they never did. [...] I think definitely somebody should look into it because I think they prefer not to rock the boat, that is the attitude that I've 
got from them. I'm totally disappointed in them (Gaby).

Le fait d'avoir vécu une telle expérience n'est pas anodin pour chacune des plaignantes. Chacune en est sortie marquée, transformée, certaines plus douloureusement que d'autres. Mais la déception face à l'efficacité des politiques, si elle est partout récurrente, est encore plus remarquable face aux organismes publics des droits de la personne, justement parce que c'est là que les plaignantes se seraient attendues à obtenir le plus de soutien et une réponse rapide et adéquate.

\section{Ce que l'analyse a révélé}

\section{Politiques à l'étude : relativement complètes}

On a vu que les politiques retenues pour l'analyse correspondent, de façon générale, au modèle qui retient les principaux éléments jugés essentiels par les auteures et auteurs comme constituant une politique efficace.

Puisque c'est le cas, puisque les enquêtes ont, de plus, démontré hors de tout doute que le harcèlement sexuel était bel et bien

«...on en est encore à percevoir le harcèlement sexuel comme un problème de femmes et non un problème impliquant l'ensemble des travailleurs.» présent en milieu de travail, et de façon persistante, comment se fait-il qu'il n'y ait pas davantage de plaintes? Non pas que l'on pense qu'il doive y avoir autant de plaintes qu'il y a d'incidents de harcèlement - une proportion d'entre eux se règle probablement à l'amiable entre les parties et n'accède donc pas à l'étape de la plainte; même en prenant ce fait en considération, il nous apparaît malgré tout que l'écart entre le nombre probable d'incidents qui nécessiteraient une plainte et le nombre réel de plaintes déposées n'est pas expliqué.

C'est du côté de la mise en application des politiques, de leur utilisation effective, qu'il faut désormais regarder, et ce, en fonction du contexte social général et organisationnel dans lequel elles sont appliquées. À cet égard, on a vu qu'il existe un certain nombre d'ambiguités et même de contradictions dans le discours des agents. 
À travers ces contradictions on s'aperçoit qu'on en est encore à percevoir le harcèlement sexuel comme un problème de femmes et non un problème impliquant l'ensemble des travailleurs ou même l'entreprise elle-même.

\section{Discours des agents : contradictions révélatrices}

Une première contradiction est évidente lorsque la majorité des agents font état de l'impact positif qu'a généré la politique. Ils affirment, d'un côté, que les hommes acceptent plus facilement les femmes dans leur milieu de travail, alors que de l'autre, ils expliquent que les hommes se sont sentis menacés lors de l'implantation de la politique et qu'encore aujourd'hui, ils ne l'apprécient guère. On peut penser que cela va nettement au delà d'une simple question d'affichage. C'est d'une attitude générale qu'il s'agit ici : on accepte mieux les femmes dans la seule mesure où elles ne dérangent rien, où elles n'exigent pas que les habitudes et les comportements soient remis en question.

Une deuxième contradiction se fait jour lorsque plusieurs agents (7) vantent la rigueur et l'efficacité de leur politique mais disent dans le même élan que les femmes ne déposent pas de plaintes parce qu'elles ont peur de devenir «visibles », ce qui implique des répercussions graves. Si la politique était réellement ce qu'ils croient, les victimes se sentiraient libres de faire valoir leurs droits, elles se sentiraient protégées et elles n'auraient pas peur.

Troisièmement, comment ne pas relever cette affirmation de dix agents selon laquelle tous les employés connaissent la politique, mais qu'il est «normal » (selon six d'entre eux) que les femmes la connaissent mieux, puisque ce sont elles qui sont susceptibles d'avoir des « problèmes $"$ ? Cette représentation des femmes comme victimes potentielles n'avalise-t-elle pas symboliquement un comportement masculin - répréhensible certes - mais quand même attendu?

Une quatrième contradiction émerge de leur discours lorsque la moitié des agents présentent l'attitude des collègues des plaignantes comme généralement positive alors qu'en réalité, les plaignantes (au dire de certains d'entre eux) peuvent s'attendre à 
«... le rôle social

d'une politique ne

semble tout

simplement pas

reconnu.» des rétroactions négatives lors de leur retour en milieu de travail après un dépôt de plainte. En fait, on peut se demander comment les agents pourraient bien connaitre l'état des rapports entre les plaignantes et leurs collègues, après la résolution de la plainte, si aucun suivi n'est effectué?

Ces contradictions font état d'une socialisation typiquement masculine : il y a des attitudes, des situations qui ne leur apparaissent tout simplement pas problématiques ou discutables. On ne parle pas ici de "mauvaise foi » ou de «mauvaise volonté »; on n'accuse pas les agents de ne pas faire correctement leur travail. Simplement, il y a comme une zone grise entre leur compréhension des situations et la mise en application de leur instrument qu'est la politique : le rôle social d'une politique ne semble tout simplement pas reconnu.

\section{Discours des plaignantes : une juste perception du pouvoir?}

Du côté des plaignantes, les témoignages recueillis soulèvent plusieurs éléments qui permettent d'entrevoir ce qui se passe réellement dans le milieu de travail et ce qui pourrait améliorer la situation de façon tangible, à l'intérieur des structures existantes. Leur discours a d'abord démontré qu'elles ont toutes eu, en général, un rapport quelconque avec la hiérarchie du pouvoir dans la société - ce qui a justifié le fait qu'elles ont choisi d'attendre longtemps avant de parler (des années, pour deux d'entre elles) ou tout simplement de ne pas rompre le silence aux premières offenses. Elles ont aussi, par exemple, fait voir que : dans le milieu de travail, l'atmosphère sexiste générale des relations amène une distorsion de départ - pour vivre une solidarité avec les collègues de travail, il faut percevoir les situations, les gestes, les rapports de la même façon qu'eux, sans quoi il est inutile de vouloir se faire comprendre - que les politiques ne possèdent pas de clause précise sur la protection des victimes contre les représailles, ni pendant le traitement de la plainte (lorsque c'est connu), ni lors du retour dans le milieu; que les victimes qui déposent une plainte sont souvent soumises à un étiquetage social au sein de leurs groupes de travail, aussi craignent-elles d'être perçues comme " traîtres » ou " plaignardes ". 
En ce qui concerne les politiques comme telles, elles ont souligné non seulement que les sanctions ne sont pas toujours précises, mais que celles qui existent sont rarement appliquées ou alors avec peu de conviction; que la définition du harcèlement sexuel reste imprécise - il faudrait reconnaître les deux types de harcèlement sexuel dans la politique pour que la victime puisse juger de l'état sérieux de sa situation en vertu de la loi; que la possibilité d'avoir le choix de se plaindre à une femme plutôt qu'à un homme faciliterait la démarche de la victime, mais elle est rarement offerte.

Finalement, en ce qui a trait aux autres lieux où la victime devrait pouvoir être entendue : les délais de traitement d'une plainte sont souvent longs (durée moyenne de deux ans à la CCDP) et la plaignante doit sans cesse faire des pressions pour que son dossier soit à l'étude. Les coordonnées (adresse, numéro de téléphone, etc.) ni des agents ni des agences fédérales concernées par la question ne figurent dans les politiques. Souvent, les plaignantes ne savent pas où aller si elles ne veulent pas faire affaire avec l'administration de leur compagnie (lorsque par exemple, l'offenseur est précisément quelqu'un de l'administration). Et, comme les plaintes peuvent être traitées à différents niveaux (à l'administration, au syndicat du niveau local ou national, à un organisme public), cela peut porter à confusion du point de vue de la plaignante et apparaitre comme un processus qui semble confus ou complexe, ce qui n'en facilite pas, là encore, le dépôt.

\section{Questions de fond}

L'ensemble de ces résultats nous permet de voir que les rapports entre les hommes et les femmes en milieu de travail sont à interpréter à partir de deux perspectives. D'une part, il y a le contexte de fond, la couleur même de l'atmosphère, si l'on peut dire, qui est teinté de sexisme et qui tend à discriminer le groupe des femmes plutôt qu'une femme en particulier. D'autre part, il y a les incidents de harcèlement envers les personnes qui se produisent sur cette toile de fond. 
Après l'analyse de ces discours, il semble évident que les causes du harcèlement sexuel et sexiste sont attribuables à la collusion de deux cultures socio-historiques: (1) la culture sociétale patriarcale, où les hommes sont socialisés en des êtres de pouvoir et de domination et (2) la culture organisationnelle du marché du travail qui est basée sur le pouvoir vertical avec des personnes en position de force. Dans un milieu de travail traditionnellement masculin, les rapports de sexe et les rapports verticaux se conjuguent: les femmes sont généralement au bas de l'échelle et peu en position de pouvoir. Elles en subissent doublement les conséquences. Et les effets d'un tel contexte sont graves tant pour les femmes comme groupe, que pour la société dans son ensemble. Que peut attendre une société d'individus qui ne peuvent utiliser leur plein potentiel au travail comme ailleurs? Quel est le coût social de la destruction psychologique d'une catégorie de personnes? Qu'on pense au discours des plaignantes!

\section{Conclusion}

Cette recherche a remis en question l'explication habituellement avancée pour rendre compte de l'écart qui existe entre le nombre de plaintes et la prévalence du harcèlement sexuel en milieu de travail traditionnellement masculin :la non efficacité des politiques implantées dans ces milieux. Elle a permis de mettre plusieurs choses en évidence.

De la même manière que la documentation avait fait voir la volonté politique des gouvernements fédéral et provincial de reconnaître le harcèlement sexuel comme problème social — ce qui leur a fait développer des lois pour tenter d'y remédier -, du côté du discours des agents, on a pu remarquer que, dans l'ensemble, on y fait preuve d'une certaine volonté en ce qui a trait à l'élimination du harcèlement sexuel en milieu de travail, mais sans exiger pourtant des sanctions précises et appropriées qui auraient un réel impact sur les attitudes masculines. Les 
contradictions identifiées dans leur discours est venu réaffirmer que le harcèlement sexuel et sexiste qui perdure est fonction du contexte organisationnel masculin qui se perpétue dans une symbolique des rapports sexués de pouvoir, tout comme certaines auteures et auteurs, notamment des féministes, l'avaient soutenu (Fitzgerald, Drasgow, Hulin, Gelfand et Magley, 1997; Sev'er, 1999; Ragins et Scandura, 1995;VanRoosmalen et McDaniel, 1998).

On en arrive donc à penser que la solution au harcèlement sexuel en milieu de travail n'est pas réductible à une question de politiques qui ne sont pas assez exhaustives, mais est plutôt liée à la mise en application de celles qui existent, et à la perception souvent étroite - de ceux qui sont responsables de son application, particulièrement dans un contexte organisationnel comme celui d'entreprises traditionnellement masculines. Mais des progrès ont été accomplis en quinze ans de recherche sur la question. On n'en est plus à douter de l'existence du phénomène dans tous les milieux de travail, ni à se poser des questions sur la nécessité d'instaurer des politiques : celles-ci sont essentielles, elles sont les pierres de soutènement sur lesquelles repose la possibilité d'action. Cependant on doit penser qu'en tant que société, il faut déployer nos efforts au-delà des politiques. On doit proposer des transformations sur le plan de leur mise en application, mais aussi de la responsabilité sociale envers le harcèlement sexuel. Cette responsabilité sociale ne peut être assumée qu'en faisant connaitre la différence qui sépare la culture masculine d'une culture sociale générale qui ne fonctionnerait pas au pouvoir, à la domination, à la hiérarchie, à l'exclusion ou à la marginalisation. Ainsi, on constate que plusieurs études sont encore nécessaires afin de cerner dans toute son ampleur la prévalence du harcèlement sexuel en milieu de travail. Une étude auprès des plaignantes comme telles s'impose nécessairement: leur perspective ajouterait à celle des agents en permettant de voir de façon beaucoup plus juste ce qui se passe en milieu de travail traditionnellement masculin, ou encore, dans divers secteurs de travail.

On pourrait maintenant faire un pas de plus et voir qu'il s'agit d'assurer non seulement une meilleure mise en application des politiques mais aussi une plus grande ouverture sociale permettant 
de faire valoir que le harcèlement sexuel, où qu'il soit, est un phénomène grave qui mérite une attention réelle.

Après l'analyse des politiques et du discours des agents, il semble que les causes du harcèlement sexuel et sexiste sont attribuables à la collusion de deux cultures socio-historiques: (1) la culture sociétale patriarcale, où les hommes sont socialisés en des êtres de pouvoir et de domination et (2) la culture organisationnelle du marché du travail qui est basée sur le pouvoir vertical avec des personnes en position de force. Dans un milieu de travail traditionnellement masculin, les rapports de sexe et les rapports verticaux se conjuguent: les femmes sont généralement au bas de l'échelle et peu en position de pouvoir. Elles en subissent doublement les conséquences. Et les effets d'un tel contexte sont graves tant pour les femmes comme groupe, que pour la société dans son ensemble. Que peut attendre une société d'individus qui ne peuvent utiliser leur plein potentiel au travail comme ailleurs? Quel est le coût social de la destruction psychologique d'une catégorie de personnes?

\section{Bibliographie}

CONNELL, Dana S. (1991a). "A Court-Mandated Sexual Harassment Policy ». Employment Relations Today, vol. 18, no 4, 433-442.

CONNELL, Dana S. (1991b). «Effective Sexual Harassment Policies: Unexpected Lessons From Jacksonville Shipyards ». Employee Relations Law Journal, vol. 17, no 2, 191-206.

CROCKER, Diane etValéry KALEMBA (1999). «The Incidence and Impact of Women’s Experiences of Sexual Harassment in Canadian Workplaces ». The Canadian Review of Sociology and Anthropology, Novembre, vol. 36, 541-558.

DRAPEAU, Maurice (1991). Le harcèlement sexuel au travail. Cowansville: Les Éditions Yvon Blais Inc.

DUPONT, Natalie (2001). Politiques contre le harcèlement sexuel: comparaison et perception des agents et des plaignantes. Thèse de M.A., Université Laurentienne, juin. Non publiée.

DUPUIS, Renée (1998). La Commission canadienne des droits de la personne et les plaintes de harcètement sexuel. Rapport final remis à la Commission canadienne des droits de la personne. Ottawa: Commission canadienne des droits de la personne, mai.

FITZGERALD, Louise F., DRASGOW, Fritz, HULIN, Charles L., GELFAND, Michele J. et Vicki J. MAGLEY (1997). «Antecedents and Consequences of Sexual Harassment in Organizations:A Test of an Integrated Model ». Journal of Applied Psychology, vol. 82, no 4, 578-589.

FLAXMAN, Howard R. et Brian F.JACKSON (1994). « New Considerations for Hostile Working 
Environment ». HR Focus, mars, vol. 71, no 3, 18-19.

GANZEL, Rebecca (1998). «What Sexual Harassment Really Prevents ». Training, October, vol. 35, no $10,86-94$.

GAUVIN, Monique (1991). «Le harcèlement sexuel et sexiste comme pratique d'appropriation des femmes: la situation dans les universités canadiennes ". Égalité. Revue acadienne d'analyse politique, printemps, 189-233.

GAUVIN, Monique, MARCOCCIO, Katherine et Alice GUÉRETTE-BREAU (1999). Le harcèlement sexiste, le harcèlement sexuel et l'agression sexuelle à l'Université de Moncton. Rapport du sondage mené auprès des étudiantes et des employées au centre universitaire de Moncton. Moncton: Groupe de recherche et d'intervention sur le harcèlement sexuel et sexiste en milieu d'enseignement francophone, février.

GIBSON, Paul C. et Kathryn PISCITELLI (1995). Sexual Harassment Manual for Managers and Supervisors. How to Prevent and Resolve Sexual Harassment Complaints in the Workplace. Second Edition, Chicago, IL: CCH Incorporated.

GUTEK, Barbara A. et Mary P. KOSS (1993). «Changed Women and Changed Organizations: Consequences of and Coping with Sexual Harassment ". Journal of Vocational Behavior, vol. 42, $28-48$.

LIPPEL, Katherine et Diane DEMERS (1998). L'accès à la justice pour des victimes de harcèlement sexuel: l'impact de la décision Béliveau-St-Jacques sur les droits des travailleuses à l'indemnisation pour les dommages. Condition féminine Canada, mars.

MATUSEWITCH, Eric (1998). «Developing and Publicizing Effective Sexual Harassment Policies». Andrews Sexual Harassment Litigation Reporter, Commentary, November $5^{\text {th }}$.

RAGINS, Belle Rose et Terri A. SCANDURA (1995). "Antecedents and Work-Related Correlates of Reported Sexual Harassment: An Empirical Investigation of Competing Hypotheses ». Sex Roles, vol. 32, nos 7-8, 429-455.

ROWE, Mary P. (1990). «People Who Feel Harassed Need a Complaint System With Both Formal and Informal Options ». Negociation Journal, avril, vol. 6, no 2,1-12.

SAVOIE, Dominique etViateur LAROUCHE (1988). «Le harcèlement sexuel au travail. Définition et mesure du phénomène ». Relations industrielles, vol. 43, no 3, 509-528.

SEV'ER, Aysan (1999). "Sexual Harassment:Where We Were, Where We Are and Prospects for the New Millennium. Introduction to the Special Issue ». The Canadian Review of Sociology and Anthropology, novembre, vol. 36, no 4, 469-497.

SMITH, Deborah (1995). «Sexual Harassment in the Workplace.The Silent Oppression ». The Social Worker/Le Travailleur Social, Summer/été, vol. 63, no 2, 85-88.

STANTON, Danielle (1992). «Harcèlement sexuel: alors on fait quoi? ». La Gazette des femmes, vol. 14, no 1, 13-20.

TERPSTRA, David E. et Douglas D. BAKER (1988). « Outcomes of Sexual Harassment Charges ». Academy of Management Journal, vol. 31, no 1, 185-194.

THACKER, Rebecca A. (1994). "Innovative Steps to Take in Sexual Harassment Prevention ». Business Horizons, janvier-février, vol. 37, 29-32.

VAN ROOSMALEN, Erica et Susan A. MCDANIEL (1998). "Sexual Harassment in Academia :A Hazard to Women's Health ». Women and Health, vol. 28, no 2, 33-54. 
WEEKS, Elaine L., BOLES, Jacqueline M., GARBIN, Albeno P. et John BLOUNT (1988). "The Transformation of Sexual Harassment from Private Trouble into a Public Issue ». Sociological Inquiry, vol. 54, no 2, 432-455.

WELSH, Sandy, DAWSON, Myrna et Elizabeth GRIFFITHS (1999a). Les plaintes de harcèlement sexuel déposées auprès de la Commission canadienne des droits de la personne. Condition féminine Canada, septembre. [document informatisé] www.swc-cfc.gc.ca/publish/research/chra-welshf.pdf.

WELSH, Sandy et Annette NIEROBISZ (1997). «How Prevalent is Sexual Harassment:A Research Note on Measuring Sexual Harassment in Canada ». Canadian Journal of Sociology, automne, vol. 22, no 4, 505-522.

\section{Notes}

1. Cette recherche a été effectuée dans le cadre du programme de maitrise en Recherches sociales appliquées , département de sociologie, Université Laurentienne, Sudbury. La thèse a été réalisée par N. Dupont sous la direction de C. Bernier et a obtenu la Médaille d'or académique de la Gouverneure générale pour excellence en recherche au niveau des études supérieures, en juin 2001. Le présent texte est une collaboration.

2. Pour préserver l'anonymat de la seule agente, dans le présent document, nous référons aux "agents » masculins.

3. Les entrevues auprès des agents sont codifiées de la façon suivante:Ag01...Ag12 sans distinction spécifique selon l'affiliation, afin de ne pas permettre leur identification (par exemple, patronal ou syndical, type de poste ou sexe). 